Virginia Commonwealth University vCU Scholars Compass

2017

\title{
A preliminary longitudinal study of white matter alteration in cocaine use disorder subjects
}

\author{
Liangsuo Ma \\ VirginiaCommonwealth University, Liangsuo.ma@vcuhealth.edu \\ Joel L. Steinberg \\ Virginia Commonwealth University \\ Qin Wang \\ Virginia Commonwealth University \\ See next page for additional authors
}

Follow this and additional works at: http://scholarscompass.vcu.edu/radiology_pubs

Part of the Radiology Commons

(C) 2017 Elsevier B.V. All rights reserved.

\section{Downloaded from}

http://scholarscompass.vcu.edu/radiology_pubs/6

This Article is brought to you for free and open access by the Dept. of Radiology at VCU Scholars Compass. It has been accepted for inclusion in Radiology Publications by an authorized administrator of VCU Scholars Compass. For more information, please contact libcompass@vcu.edu. 
Authors

Liangsuo Ma, Joel L. Steinberg, Qin Wang, Joy M. Schmitz, Edward L. Boone, Ponnada A. Narayana, and F. Gerard Moeller 
Full length article

\title{
A preliminary longitudinal study of white matter alteration in cocaine use disorder subjects
}

\author{
Liangsuo Ma ${ }^{\mathrm{a}, \mathrm{b}, *}$, Joel L. Steinberg ${ }^{\mathrm{a}, \mathrm{c}}$, Qin Wang ${ }^{\mathrm{d}}$, Joy M. Schmitz ${ }^{\mathrm{e}}$, Edward L. Boone ${ }^{\mathrm{d}}$, \\ Ponnada A. Narayana ${ }^{\mathrm{f}}$, F. Gerard Moeller ${ }^{\mathrm{a}, \mathrm{c}, \mathrm{g}, \mathrm{h}}$ \\ a Institute for Drug and Alcohol Studies, Virginia Commonwealth University (VCU), Richmond, VA, USA \\ ${ }^{\mathrm{b}}$ Department of Radiology, VCU, Richmond, VA, USA \\ ${ }^{\mathrm{c}}$ Department of Psychiatry, VCU, Richmond, VA, USA \\ d Department of Statistical Sciences and Operations Research, VCU, Richmond, VA, USA \\ e Department of Psychiatry and Behavioral Sciences, University of Texas Health Science Center (UTHSC), Houston, TX, USA \\ ${ }^{\mathrm{f}}$ Department of Diagnostic and Interventional Imaging, UTHSC, Houston, TX, USA \\ ${ }^{\mathrm{g}}$ Department of Pharmacology and Toxicology, VCU, Richmond, VA, USA \\ h Department of Neurology, VCU, Richmond, VA, USA
}

\section{A R T I C L E I N F O}

\section{Article history:}

Received 12 October 2016

Received in revised form

29 November 2016

Accepted 13 December 2016

Available online 30 January 2017

\section{Keywords:}

Diffusion tensor imaging

Cocaine use disorder

TBSS

Longitudinal design

\begin{abstract}
A B S T R A C T
Background: Previous diffusion tensor imaging (DTI) studies have consistently shown that subjects with cocaine use disorder (CocUD) had altered white matter microstructure in the corpus callosum. It is believed that these alterations are due to preexisting factors, chronic cocaine use, or both. However, there is no published longitudinal DTI study on human cocaine users yet which could shed light on the relationship between cocaine use and DTI findings.

Methods: This study used a longitudinal design and DTI to test if the white matter microstructure shows quicker alteration in CocUD subjects than controls. DTI data were acquired from eleven CocUD subjects who participated a treatment study and eleven non-drug-using controls at baseline (Scan 1) and after ten weeks (Scan 2). The baseline fractional anisotropy (FA), a general measure of white matter microstucture, and the change in FA ( $\triangle F A$, equals Scan 1 FA minus Scan $2 F A$ ) were both compared between groups. Results: The two groups did not show a difference in FA at baseline. The CocUD subjects had significantly greater $\triangle F A$ than the controls in the left splenium of the corpus callosum. In CocUD subjects, greater $\triangle F A$ in this region was associated with shorter lifetime cocaine use and greater number of positive cocaine urine samples collected during the treatment.

Conclusion: The finding in the left splenium is consistent with previous animal studies and provide indirect evidence about the effects of chronic cocaine use on white matter alterations. The subject sample size is small, therefore the results should be treated as preliminary.
\end{abstract}

(c) 2017 Elsevier B.V. All rights reserved.

\section{Introduction}

Diffusion tensor imaging (DTI), which exploits the directionality of diffusion of water molecules in tissues, is a reliable technique for non-invasively investigating the microstructure of white matter (Taber et al., 2002; Pfefferbaum et al., 2003). Though there exist exceptions (e.g., Kelly et al., 2011), the majority of previous DTI studies (Moeller et al., 2005, 2007; Lim et al., 2002, 2008; Ma et al.,

\footnotetext{
* Corresponding author at: Institute for Drug and Alcohol Studies, Department of Radiology, Virginia Commonwealth University, 203 E Cary Street, Suite 202, Richmond, VA 23219, USA.

E-mail address: Liangsuo.ma@vcuhealth.edu (L. Ma).
}

2009, 2015a; Lane et al., 2010; Romero et al., 2010; Xu et al., 2010; Bell et al., 2011; Nakamura-Palacios et al., 2016; Yip et al., 2016) have reproducibly shown that cocaine use disorder (CocUD) is associated with white matter alterations, with consistent findings in the corpus callosum (Moeller et al., 2005, 2007; Ma et al., 2009, 2015a; Lane et al., 2010; Xu et al., 2010; Bell et al., 2011). The white matter alterations found in CocUD may be related to impulsivity (Moeller et al., 2005), decision-making (Lane et al., 2010), treatment outcomes (Xu et al., 2010), or abstinence duration (Bell et al., 2011).

It is believed that the white matter alterations found in human CocUD subjects are preexisting before the onset of drug use, due to chronic drug use, or both (Narayana et al., 2009). However, our literature search did not find any published human longitudinal 
DTI studies showing the effects over time of cocaine use on white matter microstructure. The human studies reviewed above all used cross-sectional design (i.e., comparing patients with controls at one time point), and therefore cannot determine if the white matter alterations found in these studies were caused by chronic cocaine use or not. Animal studies (Narayana et al., 2009; Narayana et al., 2014) have been conducted in order to answer this question indirectly. These animal studies (Narayana et al., 2009; Narayana et al., 2014) found that the rats receiving cocaine daily for four weeks had white matter differences in corpus callosum (especially splenium of corpus callosum) compared to saline treated rats.

In light of previous animal studies and human cross-sectional studies, this preliminary study used a longitudinal design and DTI to test if the white matter microstructure is relatively more altered over time in CocUD subjects than non-drug-using controls. Eleven CocUD subjects who participated a treatment study and eleven non-drug-using controls were scanned at baseline and after ten weeks. We hypothesized that within this ten-week time frame, changes in white matter would be related to drug use in cocaine users.

\section{Methods}

\subsection{Subjects}

This study was approved by the local university Committee for the Protection of Human Subjects (CPHS) and was performed in accordance with the Code of Ethics of the World Medical Association (Declaration of Helsinki). Informed consent was obtained from each subject before being included in this study.

Treatment-seeking CocUD subjects and healthy controls were recruited via newspaper advertisements and were initially screened by a brief telephone interview. Following the phone screen, eligible subjects attended an in-person intake assessment session, in which they were screened for psychiatric disorders using the Structured Clinical Interview for DSM-IV (SCID-IV-TR) (First et al., 2002), and completed a medical history and physical examination. Information about each participant's demographic and drug use history was also collected at the intake interview. For all subjects, the Addiction Severity Index (McLellan et al., 1992) was obtained to document lifetime drug and alcohol use. Immediately prior to MRI scanning, a urine sample was obtained from each subject to screen for tetrahydrocannabinol, opiates, cocaine, amphetamines, benzodiazepines, and pregnancy (for females only). Each subject was also screened for recent alcohol use by measuring breath alcohol concentration.

Subject inclusion criteria were: (1) 18-55 years old; (2) free of alcohol at the time of magnetic resonance imaging (MRI) scanning; (3) CocUD subjects met criteria for current cocaine dependence as determined by Structured Clinical Interview for DSM-IV (SCID) (First et al., 1996); and (4) non-drug using controls had no current or lifetime history of any DSM-IV substance use or psychiatric disorder. Exclusion criteria were: (1) met current or past DSM-IV Axis I disorder other than substance abuse or dependence; (2) taking medication or having disorders that could affect the central nervous system; (3) claustrophobia during MRI simulator sessions; (4) having any definite or suspected clinically-significant brain abnormalities on the Fluid-Attenuated Inversion Recovery (FLAIR) MRI scans; (5) positive urine drug screen (for controls only); and (6) positive pregnancy test result (for females only).

Based on the inclusion and exclusion criteria, 11 CocUD subjects (CocUD group) and 11 non-drug-using controls (control group) were included for final analysis. Please see Table 1 for the age, sex, handedness, education, lifetime duration of cocaine use (years), lifetime alcohol use $(\mathrm{kg})$, and severity of cocaine dependence as measured by KMSK (Kellogg et al., 2003). The two groups did not differ significantly in age $(t=1.34$, degree of freedom $[\mathrm{df}]=20$, $\mathrm{p}=0.20)$, and years of education $(\mathrm{t}=0.73, \mathrm{df}=20, \mathrm{p}=0.48)$. Fisher's exact tests revealed that the two groups did not differ significantly in sex (2-tail $\mathrm{p}=1.00$ ) and handedness (2-tail $\mathrm{p}=1.00$ ). The between-group difference in lifetime alcohol use was trend toward significance $(\mathrm{t}=2.00, \mathrm{df}=20, \mathrm{p}=0.06)$. All CocUD subjects had DSM-IV diagnoses of current cocaine dependence and past cocaine dependence. Please see Table 1 for additional DSM-IV diagnoses of the subjects in the two groups.

\subsection{Treatment}

All the CocUD subjects also participated a treatment study (Schmitz et al., 2014), which spanned 16 weeks. During the treatment study, each CocUD subject could have received placebo, modafinil, naltrexone, l-dopa, or d-amphetamine. There were three subject visits (Monday, Wednesday, and Friday) per week. Urine samples were collected during each visit. The total number of positive cocaine urine samples per subject during the treatment period (mean \pm standard deviation) was $26.0 \pm 9.2$ (range 15-43) and the total number of negative urine samples per subject during the treatment period was $6.3 \pm 7.8(0-25)$. The negative urine samples occupied $17.9 \% \pm 19.9 \%$ of total urine samples, and three CocUD subjects provided 0 negative urine samples.

\subsection{Longitudinal study design}

For each subject, there were two MRI scanning sessions. The second scanning session (Scan 2) occurred approximately 10 weeks after the first scanning session (Scan 1). There was no significant difference $(\mathrm{t}=0.64, \mathrm{df}=20, \mathrm{p}=0.53)$ in the time interval $(\Delta \mathrm{T})$ between Scan 1 and Scan 2 between the CanUD group ( $72.0 \pm 13.1$ days) and the control group $(67.5 \pm 19.3$ days). For the CocUD subjects, Scan 1 occurred $10.2 \pm 8.4$ days before the initialization of the treatment. Therefore there was a mismatch between the timing of the treatment-related urine drug testing and the time between the two MRI scanning sessions.

\subsection{MRI data acquisition}

For each scanning session (Scan 1 or Scan 2), MRI data were acquired on a Philips 3.0T Intera system with a six channel receive head coil (Philips Medical Systems, Best, Netherlands). Whole brain diffusion-weighted images (DWI) were acquired in the transverse plane using a single shot diffusion sensitized spin echo echo-planar imaging (EPI) sequence, with the following parameters: bfactor $=1000 \mathrm{~s} / \mathrm{mm}^{2}$, repetition time $=6100 \mathrm{~ms}$, echo time $=84 \mathrm{~ms}$, 44 contiguous axial slices, field-of-view $=240 \mathrm{~mm} \times 240 \mathrm{~mm}$, $112 \times 112$ acquisition matrix, $256 \times 256$ reconstructed matrix, $0.9375 \mathrm{~mm} \times 0.9375 \mathrm{~mm}$ reconstructed in-plane resolution, slice thickness $=3 \mathrm{~mm}$, and zero interslice gap. The diffusion tensor encoding scheme was based on the uniformly distributed and balanced rotationally invariant Icosa21 (21 gradient directions) tensor-encoding set (Hasan and Narayana, 2003). A SENSE acceleration factor of 2 was used for the DWI acquistion. The diffusion-encoded volumes were acquired with fat suppression. The DTI acquisition time was approximately $7 \mathrm{~min}$. FLAIR scans and T2-weighted spin-echo scans were acquired in Scan 1 and were read by a board-certified radiologist in order to rule-out any incidental brain pathology.

\subsection{DTI data processing}

The DTI images in each scanning session were processed using the FMRIB Software Library (FSL) (www.fmrib.ox.ac.uk/fsl, ver- 
Table 1

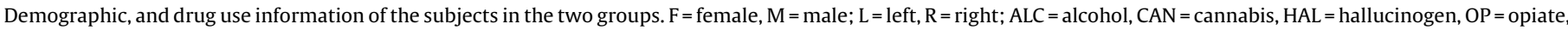
$\mathrm{PCP}=$ phencyclidine, $\mathrm{SED}=$ sedative, $\mathrm{STIM}=$ stimulant, $\mathrm{XTC}=$ ecstasy; $\mathrm{AB}=$ abuse, $\mathrm{DEP}=$ dependence, $\mathrm{SD}=$ standard deviation, $\mathrm{N} / \mathrm{A}=$ not applicable.

\begin{tabular}{|c|c|c|}
\hline Parameter & $\operatorname{CocUD}(\mathrm{n}=11)$ & Control $(\mathrm{n}=11)$ \\
\hline Age mean \pm SD (years) (range) & $\begin{array}{l}42.8 \pm 6.4 \\
(26.9-50.6)\end{array}$ & $\begin{array}{l}38.7 \pm 8.0 \\
(24.0-54.1)\end{array}$ \\
\hline Sex & $2 \mathrm{~F}, 9 \mathrm{M}$ & $2 \mathrm{~F}, 9 \mathrm{M}$ \\
\hline Handedness & $1 \mathrm{~L}, 10 \mathrm{R}$ & $2 \mathrm{~L}, 9 \mathrm{R}$ \\
\hline Education mean $\pm S D$ (years) (range) & $\begin{array}{l}13.3 \pm 1.8 \\
(12-17)\end{array}$ & $\begin{array}{l}13.8 \pm 1.9 \\
(11-16)\end{array}$ \\
\hline Lifetime alcohol use mean $\pm \mathrm{SD}(\mathrm{kg})$ (range) & $\begin{array}{l}229.5 \pm 326.3 \\
(0-1130.0)\end{array}$ & $\begin{array}{l}20.1 \pm 30.6 \\
(0-100.8)\end{array}$ \\
\hline Lifetime cocaine use mean \pm SD (years) (range) & $\begin{array}{l}13.3 \pm 5.1 \\
(5-20)\end{array}$ & $\mathrm{N} / \mathrm{A}$ \\
\hline Total KMSK mean \pm SD [range] & $\begin{array}{l}11.6 \pm 2.7 \\
(7-14)\end{array}$ & $\mathrm{N} / \mathrm{A}$ \\
\hline Additional DSM-IV diagnoses & $\begin{array}{l}2 \text { current ALC AB } \\
2 \text { past ALC DEP } \\
1 \text { current CAN AB } \\
2 \text { past CAN AB } \\
1 \text { past CAN DEP } \\
1 \text { past HAL DEP } \\
1 \text { past OP AB } \\
1 \text { past PCP DEP } \\
1 \text { past SED AB } \\
2 \text { past STIM DEP } \\
1 \text { past XTC DEP }\end{array}$ & 1 past ALC $A B$ \\
\hline
\end{tabular}

sion 5.04) (Jenkinson et al., 2012). For each data set, the DWI images were corrected for eddy current distortions and head motion (Jenkinson and Smith, 2001) after converting the Philips DICOM files into NIfTI format using $\mathrm{dcm} 2$ nii as implemented in MRIcron (http://www.mccauslandcenter.sc.edu/mricro/mricron/). Next, brain was extracted from the images using FSL's Brain Extraction Tool (Smith, 2002). After these preprocessing steps, the FMRIB's Diffusion Toolbox (Behrens et al., 2003) was used to fit the data to extract the DTI parameters for each voxel. The DTI parameters included fractional anisotropy (FA), mean diffusivity (MD), the three eigenvalues (L1, L2, and L3) and the three eigenvectors (V1, V2, and V3). Among these DTI measures, FA has been most commonly used. Axial diffusivity, radial diffusivity, and MD are the other three commonly used DTI measures. The axial diffusivity $\left(\mathrm{L}_{1}\right)$ is the same as the first eigenvalue, and the radial diffusivity $\left(\mathrm{L}_{\mathrm{T}}\right)$ is calculated as the average of the second (L2) and third (L3) eigenvalues. It is commonly regarded that FA is a general measure of white matter microstucture and that axial and radial diffusivities may be more pathologically specific than FA (Hasan and Narayana, 2006).

\subsection{Tract-based spatial statistics (TBSS) voxelwise statistcal analysis}

Whole brain voxelwise statistical analysis of the DTI data was carried out using Tract-Based Spatial Statistics (TBSS) (Smith et al., 2006), part of the FSL software. The FA images from all subjects and the two scanning sessions were aligned to the standard MNI (Montreal Neurological Institute) space using the FSL's nonlinear registration with the FMRIB58_FA template image. Next, the mean FA image was created and thinned to create a mean FA skeleton representing the centers of all tracts common to all subjects. The FA threshold was set to be $F A=0.20$. Each subject's aligned FA data that was a local maximum within a short-radius plane normal to each point on the skeleton was then projected onto that point of the skeleton. The skeletonization operation was also applied to the other DTI measures (MD, $\mathrm{L}_{1}$, and $\mathrm{L}_{\mathrm{T}}$ ). For each subject, the change of FA on the skeleton between Scan 1 and Scan 2 was defined using the formula $\triangle F A=F A(S \operatorname{San} 1)-F A(S \operatorname{Scan} 2)$. Larger $\triangle F A$ between groups suggests larger white matter alteration. The changes of other DTI measures ( $\Delta \mathrm{MD}, \Delta \mathrm{L}_{1}, \Delta \mathrm{L}_{\mathrm{T}}$ ) were similarly computed.
The TBSS analysis, which tested group difference in the change of DTI measures (i.e., $\Delta \mathrm{FA}, \Delta \mathrm{MD}, \Delta \mathrm{L}_{1}, \Delta \mathrm{L}_{\mathrm{T}}$ ), was conducted voxelwise over the whole-brain skeleton using the FSL nonparametric program, Randomise, with a Threshold-Free Cluster Enhancement (TFCE) option and 10000 random permutations. TFCE (Smith and Nichols, 2009; Salimi-Khorshidi et al., 2011) is a robust clusterbased thresholding approach, without a need of arbitrary initial cluster-forming threshold. The TBSS randomise procedure automatically uses family-wise error (FWE) correction for multiple comparisons. Given the two group comparisons (CocUD $>$ CTL, CocUD $<\mathrm{CTL}$ ), statistical significance of TBSS in the Randomise analysis was defined as 2-tail $\mathrm{p}<0.05$ (FWE corrected). Because $\Delta \mathrm{FA}$ (or $\left.\Delta \mathrm{MD}, \Delta \mathrm{L}_{1}, \Delta \mathrm{L}_{\mathrm{T}}\right)$ was dependent on the time interval $(\Delta \mathrm{T})$ between Scan 1 and Scan $2, \Delta \mathrm{T}$ was included as the covariate in the TBSS analysis. The group difference in the change of DTI measures could be related to group difference in the DTI measures at baseline (Scan 1 ). In order to test this, another TBSS analysis, which tested group difference in the DTI measures (i.e., FA, MD, $\mathrm{L}_{1}$, and $\mathrm{L}_{\mathrm{T}}$ ) at baseline (Scan 1), was conducted with same settings.

The JHU DTI-based ICBM-DTI-81 WM labeled atlas (Mori et al., 2005; Wakana et al., 2007; Hua et al., 2008) was used to determine labels for brain regions that showed group differences.

\section{Results}

\subsection{TBSS DTI analysis}

The CocUD group had significantly greater $\triangle F A$ than the CTL group in one cluster (FWE corrected $\mathrm{p}<0.05,2$-tail). This cluster $(48$ voxels), which was from the "tfce_corrp_tstat" output of the "randomise" command, was in the left splenium of the corpus closum (Fig. 1). The mean (acoss all voxels in the cluster) $\triangle F A$ in this cluster was $0.0172 \pm 0.0141$ (mean \pm standard deviation) for the CocUD group and $-0.0114 \pm 0.0131$ for the control group. Fig. 2 shows individual participant FA value at each scan time for the left splenium of the corpus callosum and also for a comparison reference region right superior longitudinal fasciculus (SLF) that did not show any change from Scan 1 to Scan 2. The FA value shown in Fig. 2 was the mean FA value across all voxels in each region (splenium or SLF). The splenium region is the region showing significant group difference as shown in Fig. 1, and the SLF region was a sphere with $3 \mathrm{~mm}$ 


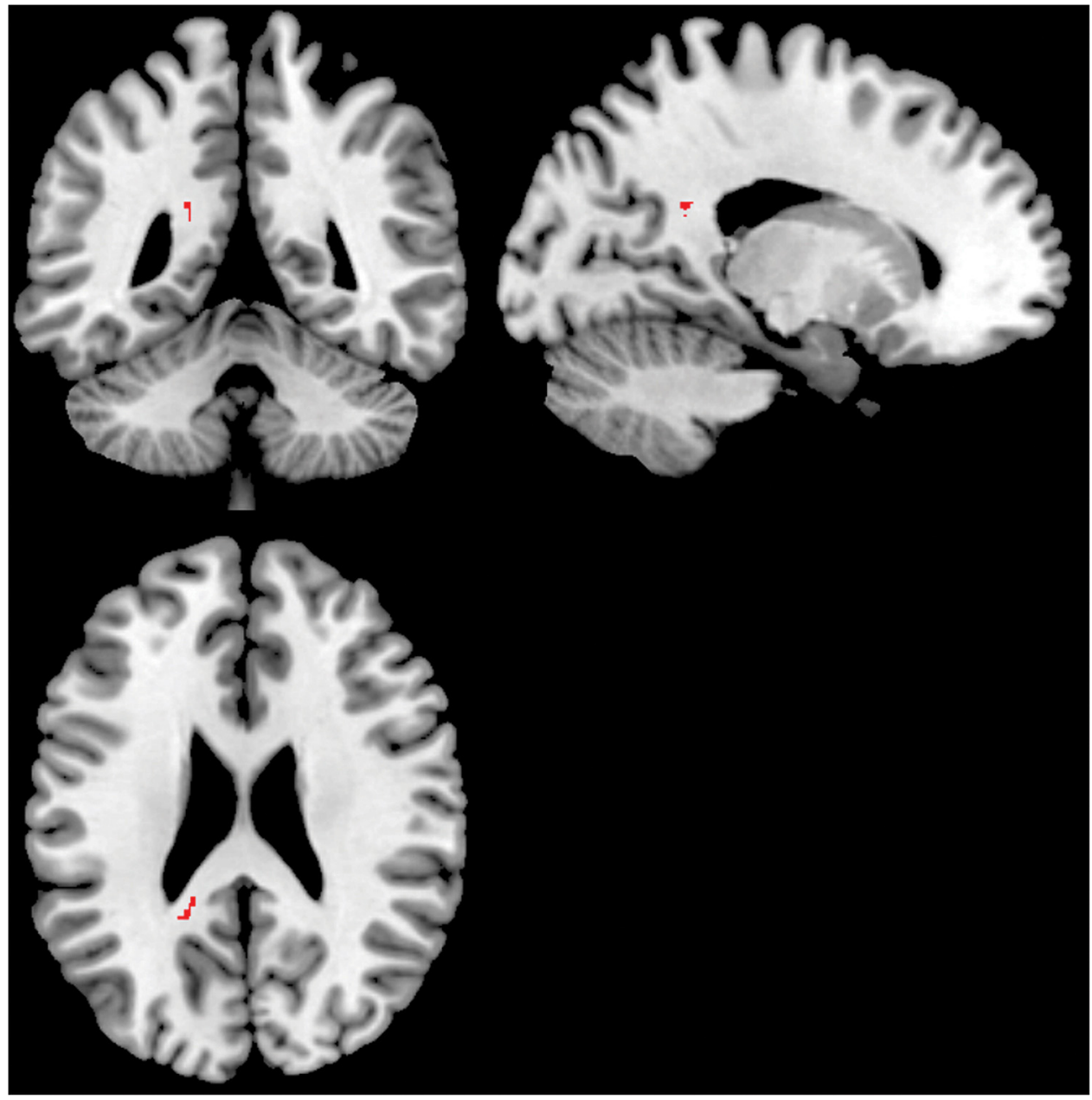

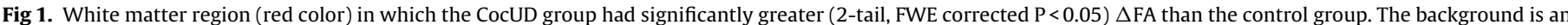

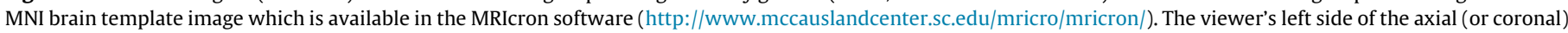

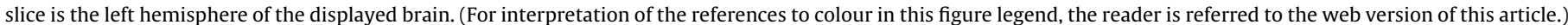
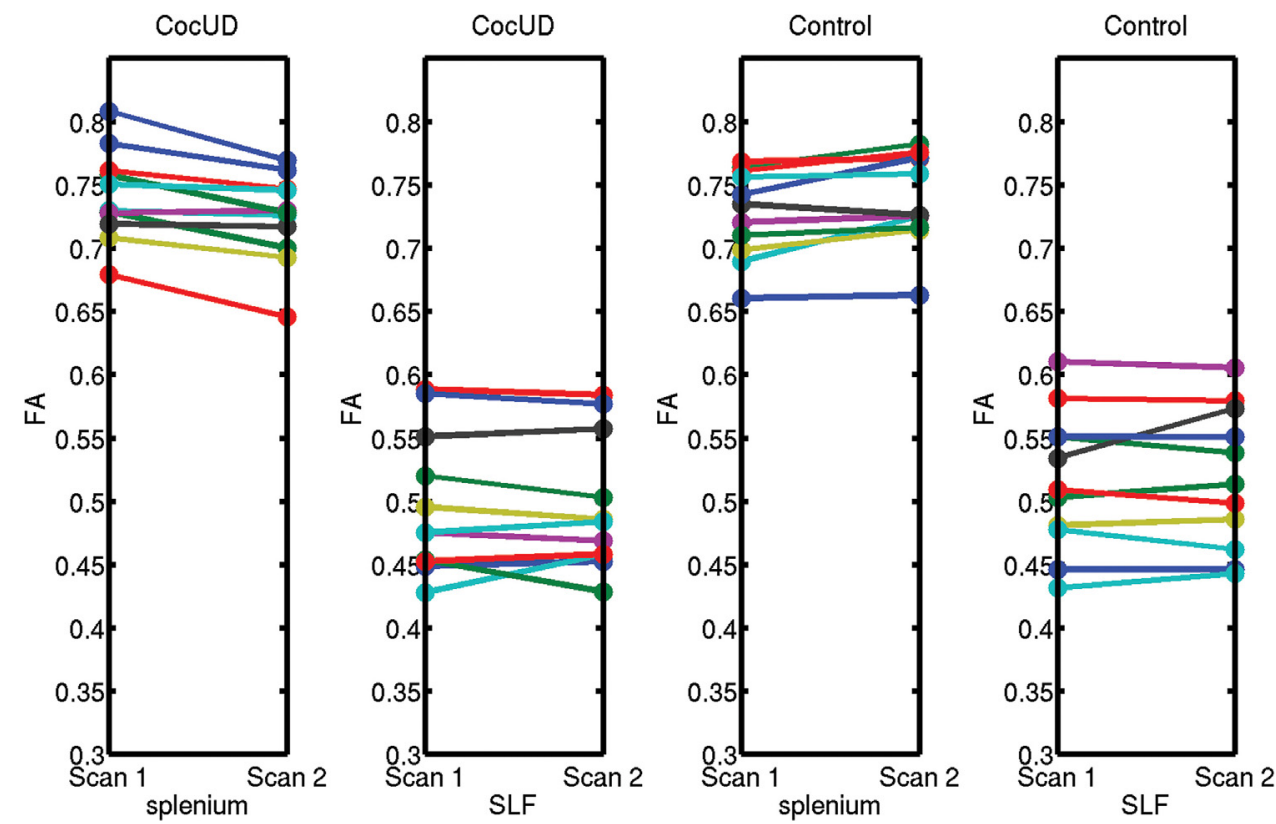

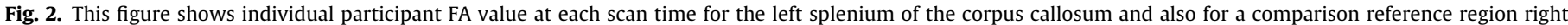

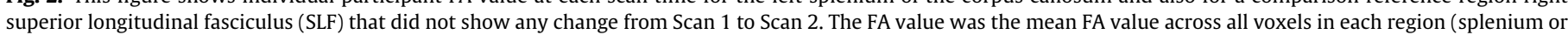

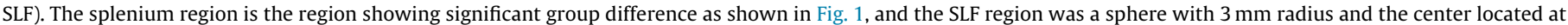
MNI coordinate of [37-23 30]. 
radius and the center located at MNI coordinate of [37-23 30]. No significant group difference was found in $\triangle F A$ for the other direction of comparison (CocUD $<\mathrm{CTL}$ ). No significant group difference was found for the other DTI measures $\left(\Delta \mathrm{MD}, \Delta \mathrm{L}_{1}, \Delta \mathrm{L}_{\mathrm{T}}\right)$ for any direction of comparison (CocUD $>$ CTL, or CocUD $<$ CTL).

In order to test within-group changes, separate within-group TBSS analyses were conducted in the CocUD group and the control group respectively. For the CocUD group, there were several clusters in which $\triangle F A$ was significant greater than zero (FWE corrected $\mathrm{p}<0.05,2$-tail). These clusters, which $100 \%$ contained the cluster found in the above between-group TBSS analysis, were in the body of corpus callosum ( 400 voxels), splenium of corpus callosum (1047 voxels), left cerebral peduncle (66 voxels), left posterior limb of internal capsule (140 voxels), left retrolenticular part of internal capsule (306 voxels), left anterior corona radiata (31 voxels), left superior corona radiata (169 voxels), left posterior corona radiata (198 voxels), right posterior corona radiata (134 voxels), left posterior thalamic radiation (includes optic radiation) (129 voxels), left sagittal stratum (includes inferior longitidinal fasciculus and inferior fronto-occipital fasciculus) (46 voxels), left external capsule (117 voxels), left superior longitudinal fasciculus (51 voxels), left uncinate fasciculus (34 voxels). No cluster was found in which $\triangle F A$ was significantly less than zero. For the control group, no cluster was found in which $\triangle F A$ was significantly greater than or significantly less than zero.

At baseline (Scan 1), no significant group difference was found in FA or other DTI measures (i.e., $M D, \mathrm{~L}_{1}$, and $\mathrm{L}_{\mathrm{T}}$ ) for both directions of comparison (CocUD $>$ CTL, or CocUD $<$ CTL).

\subsection{Relationship between $\triangle F A$ and other biological measures}

A post hoc regression analysis was conducted (using statistical software $\mathrm{R}$ version 3.2.0) in order to quantify the relationship between the mean $\Delta \mathrm{FA}$ (average across all the voxels) on the splenium of corpus callosum showing group difference and other biological measures. These measures include one of the treatment outcomes (i.e., positive urine samples collected during the treatment), lifetime cocaine use (years), lifetime alcohol use (kg), and total KMSK score (cocaine dependence severity). In order to account for the effects of $\Delta T$, and the total number of urine samples (the sum of the number of positive urine samples and the number of negative urine samples), these two measures were included in the regression analysis as covariates (factors of non-interest), with $\triangle \mathrm{FA}$ being the response in the model. Akaike information criterion (AIC) was used to select the model which fits the data best.

The regression analysis $\left(F=10.22, R^{2}=0.87\right.$, and $\left.p=0.008\right)$ found a significant positive correlation between $\triangle F A$ and the number of positive cocaine urine samples $(\mathrm{p}=0.001)$. Scatter plot between $\triangle F A$ and the number of positive cocaine urine samples in CocUD subjects is shown in Fig. 3. In addition, the lifetime cocaine use was found to be negatively correlated with $\triangle F A(p=0.01)$. Neither life time alcohol use $(p=0.89)$ nor total KMSK score $(p=0.15)$ showed significant effect on $\triangle F A$. The negative correlation between the $\triangle F A$ and the lifetime cocaine use could be due to lower baseline FA (FA at Scan 1) in the CocUD subjects with longer lifetime use (see Discussion section). Consistently, both Pearson ( $\mathrm{rho}=-0.6908$, $\mathrm{p}=0.0186$ ) and Spearman ( $\mathrm{rho}=0.6636, \mathrm{p}=0.0260$ ) correlation analyses found a significant and negative correlation between the baseline FA (Scan 1) and the lifetime cocaine use (years). Scatter plot between the baseline FA (Scan 1) and the lifetime cocaine use (years) in CocUD subjects is shown in Fig. 4.

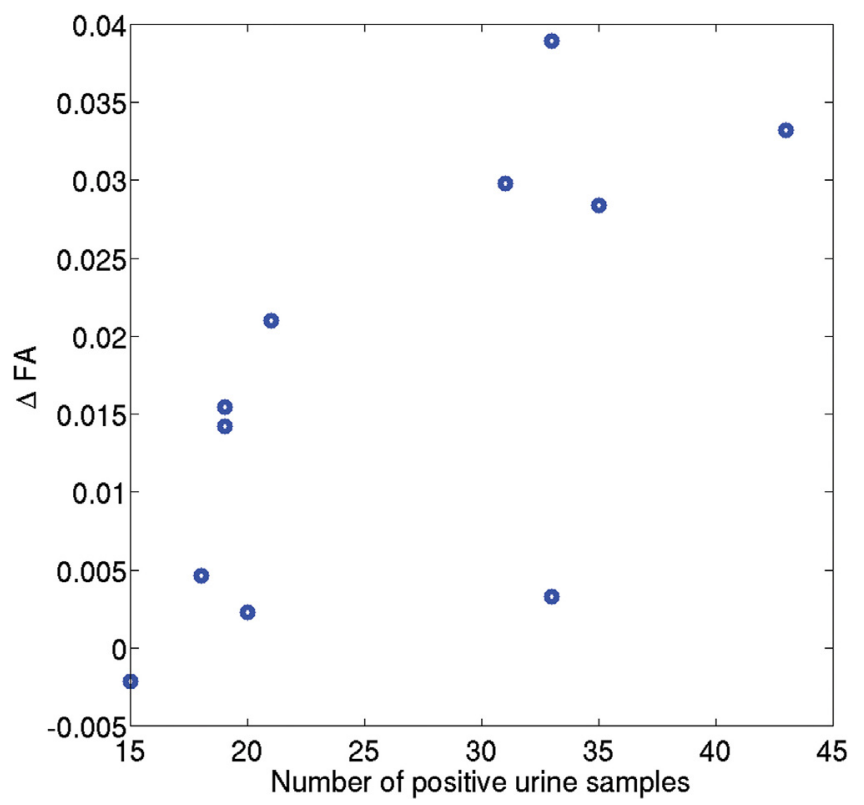

Fig. 3. Scatter plot between $\triangle F A$, which is defined as FA (Scan 1) - FA (Scan 2), and the number of positive cocaine urine samples in CocUD subjects. The regression analysis $\left(F=10.22, R^{2}=0.87\right.$, and $\left.p=0.008\right)$ found a significant positive correlation between $\triangle F A$ and the number of positive cocaine urine samples $(p=0.001)$.

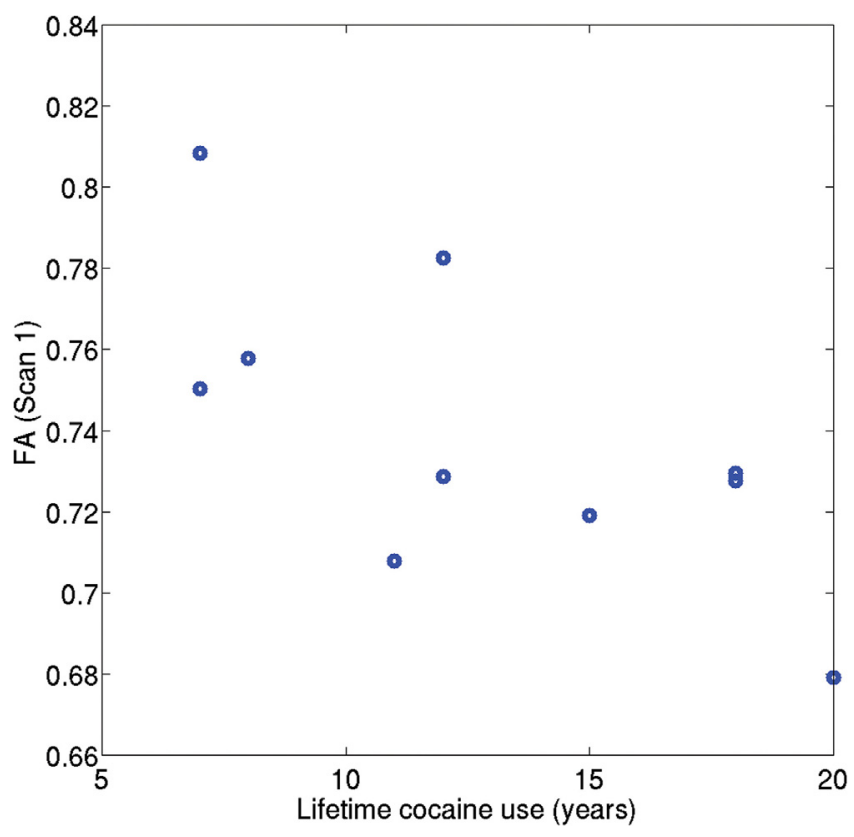

Fig. 4. Scatter plot between FA (Scan 1) and the lifetime cocaine use (years) in CocUD subjects. Both Pearson ( $r h o=-0.6908, p=0.0186$ ) and Spearman ( $r h o=0.6636$, $\mathrm{p}=0.0260$ ) correlation analyses found a significant and negative correlation between the baseline FA (Scan 1) and the lifetime cocaine use (years).

\section{Discussion}

Using a longitudinal design and DTI, we tested whether CocUD subjects had greater white matter alteration than non-drug-using control subjects in a ten-week time frame. This was achieved by testing the group difference in the change of whole brain white matter microstructure, as quantified by the changes in DTI measures, between baseline (Scan 1) and ten weeks later (Scan 2). The DTI TBSS analysis found that the FA (a measure of overall white matter microstucture) in the left splenium of corpus callosum in the CocUD subjects showed a significantly greater decrease over 
time compared to the control subjects, suggesting greater white matter alteration over time in this region in the CocUD subjects. The regression analysis indicated that in the CocUD subjects, the greater reduction of FA in this region (left splenium of corpus callosum) was associated with shorter lifetime cocaine use (years) and greater number of positive cocaine urine samples collected during the treatment.

Previous human cross sectional studies (Moeller et al., 2005, 2007; Lim et al., 2002, 2008; Ma et al., 2009, 2015a; Lane et al., 2010; Romero et al., 2010; Xu et al., 2010; Bell et al., 2011; NakamuraPalacios et al., 2016) compared DTI measures between CocUD and controls at a single time point. Thus the white matter alterations found by these studies could be due to the combination of preexisting factors and chronic cocaine use. Differently, the present study assessed the between-group difference in the change of FA over an approximately ten-week period of time. Thus the findings of the present study may be related to cocaine use during the period between Scan 1 and Scan 2 . However, the present study still did not provide direct causal relationship between cocaine use and white matter degradation, since possible effects on white matter of the medications used in the treatment period (which largely overlapped with the period between Scan 1 and Scan 2) have not been ruled out. By assessing the change of FA, some complicate factors (such as group difference in environmental effects) were cancelled out. This may account for why significant group difference was found in the change of FA but not in the FA at baseline (Scan 1).

The evidences of negative effects that chronic cocaine use exerts on white matter are largely from animal studies. Previous rodent DTI studies (Narayana et al., 2009; Narayana et al., 2014) found that rats receiving cocaine daily for four weeks had significantly lower FA than those rats daily receiving saline in the same period. The white matter change was in splenium of corpus callosum (Narayana et al., 2009; Narayana et al., 2014), genu of corpus callosum (Narayana et al., 2014), and internal capsule (Narayana et al., 2014). In addition, the significant reduction of FA was left hemisphere dominant (Narayana et al., 2014). The present study found that the FA in the left splenium of corpus callosum in the CocUD subjects showed significantly greater reduction compared to the control subjects. Thus the present human study is consistent with the published rodent studies (Narayana et al., 2009; Narayana et al., 2014), as reflected by the following two points. Firstly, the rodent studies (Narayana et al., 2009; Narayana et al., 2014) and the present study found reduced FA and greater reduced FA, respectively, in the splenium of corpus callosum. Secondly, both the Narayana et al. (2014) rodent study and the present study found reduced FA and greater reduced FA, respectively, in the left hemisphere. The consistencies between the present study and previous rodent studies (Narayana et al., 2009; Narayana et al., 2014) support the interpretation that the findings of the present study were possibly due to cocaine use during the period between Scan 1 and Scan 2.

We also tested whether the greater reduction of FA in the left splenium of corpus callosum found in the CocUD subjects was associated with other biological measures, such as the number of positive cocaine urine samples collected during the treatment, lifetime cocaine use, lifetime alcohol use, and severity of cocaine dependence. The regression analysis did not found significant relationship between the reduction of FA and the lifetime alcohol use, or severity of cocaine dependence, suggesting the effects of these two factors were neglectable. The regression analysis found that the less reduction of FA was associated with longer lifetime cocaine use (in years), suggesting that white matter degradation is less for the CocUD subjects with longer lifetime use. This may be due to the fact that white matter alteration may have happened in this region (left splenium of corpus callosum) in the subjects with longer life- time cocaine use, and there is less room for further alteration in these CocUD subjects. The negative correlation between baseline FA (FA at Scan 1) and the lifetime cocaine use (years) support this interpretation. The regression analysis also found that the greater reduction of FA was associated with the greater number of positive cocaine urine samples. As discussed above, the reduction of FA was likely due to the cocaine use in the period between Scan 1 and Scan 2 , which largely overlap the treatment in which the urine samples were collected. The positive cocaine urine samples and the reduction of FA were due to or likely due to cocaine use respectively. Thus, these two measures could be circular (Kriegeskorte et al., 2009), and the significant correlation between them does not provide further evidence for their relationship.

The results of the present study may have implications for the treatment of CocUD. The FA value in corpus callosum has been reported to be significantly and positively correlated with duration of cocaine abstinence in treatment-seeking CocUD subjects ( Xu et al., 2010). Consistently, the present study found greater reduction of FA in the splenium of corpus callosum in CocUD subjects. Thus, recovery from white matter alteration, which has the potential in improving treatment outcomes, could be a target of treatment for CocUD. A recent rodent study (Lan et al., 2015) reported that pioglitazone can significantly reduce white matter lesion in corpus callosum, caudate, putamen, external capsule, and internal capsule. Interestingly, the corpus callosum reported by Lan et al. (2015) is also found in the present study and is consistently reported to be a region showing white matter alteration in both human CocUD subjects (Moeller et al., 2005, 2007; Ma et al., 2009, 2015a; Lane et al., 2010; Xu et al., 2010; Bell et al., 2011) and cocaine exposed rodents (Narayana et al., 2009; Narayana et al., 2014). In addition, the internal capsule found by Lan et al. (2015) is also the region showing white matter alteration in the rats daily treated with cocaine (Narayana et al., 2014). Furthermore, striatal regions are part of network showing cognitive impairments in CocUD (Ma et al., 2015b, 2014).These consistencies suggest that pioglitazone has the potential in slowing down white matter alteration in CocUD individuals, especially in white matter regions which may be related to cocaine use.

There exist longitudinal DTI studies that investigated topics other than CocUD, such as head injury (Murugavel et al., 2014; Chun et al., 2015; Edlow et al., 2016), cognitive training (Engvig et al., 2012), and virus infection (Tang et al., 2016). Among these studies, Engvig et al. (2012) used TBSS to investigate changes in white matter microstructure within ten weeks, and thus is methodically similar to the present study. The present human study is consistent with Engvig et al. (2012) in several aspects. Firstly, both studies show that DTI is sensitive to alterations of white matter microstructure over an interval of ten weeks. Secondly, both studies found changes in FA. Thirdly, both studies found changes in left hemisphere only. Finally, both studies suggest potential functional/clinical significance of the changes found by the studies. Engvig et al. (2012) reported a positive effect of cognitive training on white matter microstructure as reflected by the increased FA and the significant association between greater memory improvement and greater increase of FA; correspondingly the present study found a negative effect of cocaine use on white matter microstructure as reflected by the reduced FA and the significant association between the greater number of positive urine samples and the greater reduction of FA. A difference between these two studies is that alterations in radial diffusivity and axial diffusivity were found in Engvig et al. (2012) but not in the present study.

A strength of the present study is the longitudinal design. Some of the limitations of DTI include sensitivity to motion and scanning time (Mori and Zhang, 2006), limited spatial resolution (Enzinger et al., 2015), less ability in providing information in grey matter and regions with low FA (Enzinger et al., 2015). One of these limitations, 
i.e., sensitivity to motion, is particularly relevant to the present study, which used a longitudinal design. As discussed in Engvig et al. (2012), multiple time points related variation in head motion or head placement in the scanner could confound findings of longitudinal studies. The halfway linear registration (FA images for each time-point for each subject are linearly registered to a space halfway between the first scan time and the second scan time) and smoothing step proposed by Engvig et al. (2012) could reduce this confounding effect. A limitation specific to the present study is the small sample size, and therefore the results should be treated as preliminary and interpreted with caution. The small subject sample size was partially related to the longitudinal nature which has subject drop-out over time (20 CocUD and 12 control subjects dropped after the MRI data were acquired at baseline). Another limitation specific to the present study is that the period in which the urine samples were collected did not completely coincide with the period in which the MRI data were collected. The third limitation specific to the present study is that the methods proposed by Engvig et al. (2012) were not used. Future studies addressing these limitations are warranted in order to further clarify the findings of the present study.

\section{Contributors}

All authors reviewed and edited the manuscript. Liangsuo Ma analyzed all data, and wrote up the manuscript. F. Gerard Moeller, Liangsuo Ma, Joel L. Steinberg, Joy M. Schmitz, and Ponnada A. Narayana designed the experiment. Qin Wang conducted the regression analysis. All authors have approved the final version of this manuscript.

\section{Conflict of interest}

No conflict declared.

\section{Role of funding source}

Nothing declared.

\section{Acknowledgements}

This work is financially supported by National Institute on Drug Abuse (NIDA) Grants \# R01 DA034131 (LM), P50DA009262 (JLS), U54 DA038999 (FGM), and MCRR Shared Instrumentation Grant \# 1 S10 RR019186-01 (PAN).

\section{References}

Behrens, T.E., Johansen-Berg, H., Woolrich, M.W., Smith, S.M., Wheeler-Kingshott, C.A., Boulby, P.A., Barker, G.J., Sillery, E.L., Sheehan, K., Ciccarelli, O., Thompson, A.J., Brady, J.M., Matthewset, P.M., 2003. Non-invasive mapping of connections between human thalamus and cortex using diffusion imaging. Nat. Neurosci. 6, 750-757.

Bell, R.P., Foxe, J.J., Nierenberg, J., Hoptman, M.J., Garavan, H., 2011. Assessing white matter integrity as a function of abstinence duration in former cocaine-dependent individuals. Drug Alcohol Depend. 114, 159-168.

Chun, I.Y., Mao, X., Breedlove, E.L., Leverenz, L.J., Nauman, E.A., Talavage, T.M., 2015. DTI detection of longitudinal WM abnormalities due to accumulated head impacts. Dev. Neuropsychol. 40, 92-97.

Edlow, B.L., Copen, W.A., Izzy, S., Bakhadirov, K., van der Kouwe, A., Glenn, M.B. Greenberg, S.M., Greer, D.M., Wu, O., 2016. Diffusion tensor imaging in acute-to-subacute traumatic brain injury: a longitudinal analysis. BMC Neurol 16

Engvig, A., Fjell, A.M., Westlye, L.T., Moberget, T., Sundseth, O., Larsen, V.A. Walhovd, K.B., 2012. Memory training impacts short-term changes in aging white matter: a longitudinal diffusion tensor imaging study. Hum. Brain Mapp. 33, 2390-2406.

Enzinger, C., Barkhof, F., Ciccarelli, O., Filippi, M., Kappos, L., Rocca, M.A., Ropele, S., Rovira, A., Schneider, T., de Stefano, N., Vrenken, H., Wheeler-Kingshott, C., Wuerfel, J., Fazekas, F., Grp, M.S., 2015. Nonconventional MRI and microstructural cerebral changes in multiple sclerosis. Nat. Rev. Neurol. 11, 676-686.

First, M.B., Spitzer, R.L., Gibbon, M., Williams, J.B.W., 1996. Structured Clinical Interview for DSM-IV Axis I Disorders-Patient Edition (SCID-I/P, Version 2.0) Human Brain Mapping Biometrics Research Department. New York State Psychiatric Institute, New York.

First, M.B., Gibbon, M., Spitzer, R.L., Williams, J.B.W., 2002. Structured Clinical Interview for DSM-IV-TR Axis I Disorders, Research Version, Patient Edition. Biometrics Research New York State Psychiatric Institute, New York.

Hasan, K.M., Narayana, P.A., 2003. Computation of the fractional anisotropy and mean diffusivity maps without tensor decoding and diagonalization: theoretical analysis and validation. Magn. Reson. Med. 50, 589-598.

Hasan, K.M., Narayana, P.A., 2006. Retrospective measurement of the diffusion tensor eigenvalues from diffusion anisotropy and mean diffusivity in DTI. Magn. Reson. Med. 56, 130-137

Hua, K., Zhang, J., Wakana, S., Jiang, H., Li, X., Reich, D.S., Calabresi, P.A., Pekar, J.J., van Zijl, P.C., Mori, S., 2008. Tract probability maps in stereotaxic spaces: analyses of white matter anatomy and tract-specific quantification. Neuroimage 39, 336-347.

Jenkinson, M., Smith, S., 2001. A global optimisation method for robust affine registration of brain images. Med. Image Anal. 5, 143-156.

Jenkinson, M., Beckmann, C.F., Behrens, T.E., Woolrich, M.W., Smith, S.M., 2012. Fsl. Neuroimage 62, 782-790.

Kellogg, S.H., McHugh, P.F., Bell, K., Schluger, J.H., Schluger, R.P., LaForge, K.S., Ho, A., Kreek, M.J., 2003. The Kreek-McHugh-Schluger-Kellogg scale A new, rapid method for quantifying substance abuse and its possible applications. Drug Alcohol Depend. 69, 137-150.

Kelly, C., Zuo, X.N., Gotimer, K., Cox, C.L., Lynch, L., Brock, D., Imperati, D., Garavan, H., Rotrosen, J., Castellanos, F.X., Milham, M.P., 2011. Reduced interhemispheric resting state functional connectivity in cocaine addiction. Biol. Psychiatry 69, 684-692.

Kriegeskorte, N., Simmons, W.K., Bellgowan, P.S., Baker, C.I., 2009. Circular analysis in systems neuroscience: the dangers of double dipping. Nat. Neurosci. 12, 535-540.

Lan, L.F., Zheng, L., Yang, X., Ji, X.T., Fan, Y.H., Zeng, J.S., 2015. Peroxisome proliferator-activated receptor-gamma agonist pioglitazone ameliorates white matter lesion and cognitive impairment in hypertensive rats. CNS Neurosci. Ther. 21, 410-416.

Lane, S.D., Steinberg, J.L., Ma, L., Hasan, K.M., Kramer, L.A., Zuniga, E.A., Narayana, P.A., Moeller, F.G., 2010. Diffusion tensor imaging and decision making in cocaine dependence. PLoS One 5, e11591.

Lim, K.O., Choi, S.J., Pomara, N., Wolkin, A., Rotrosen, J.P., 2002. Reduced frontal white matter integrity in cocaine dependence: a controlled diffusion tenso imaging study. Biol. Psychiatry 51, 890-895.

Lim, K.O., Wozniak, J.R., Mueller, B.A., Franc, D.T., Specker, S.M., Rodriguez, C.P., Silverman, A.B., Rotrosen, J.P., 2008. Brain macrostructural and microstructural abnormalities in cocaine dependence. Drug Alcohol Depend. 92, 164-172.

Ma, L., Hasan, K.M., Steinberg, J.L., Narayana, P.A., Lane, S.D., Zuniga, E.A., Kramer, L.A., Moeller, F.G., 2009. Diffusion tensor imaging in cocaine dependence: regional effects of cocaine on corpus callosum and effect of cocaine administration route. Drug Alcohol Depend. 104, 262-267.

Ma, L., Steinberg, J.L., Hasan, K.M., Narayana, P.A., Kramer, L.A., Moeller, F.G., 2014. Stochastic dynamic causal modeling of working memory connections in cocaine dependence. Hum. Brain Mapp. 35, 760-778.

Ma, L., Steinberg, J.L., Keyser-Marcus, L., Ramesh, D., Narayana, P.A., Merchant, R.E., Moeller, F.G., Cifu, D.X., 2015a. Altered white matter in cocaine-dependent subjects with traumatic brain injury: a diffusion tensor imaging study. Drug Alcohol Depend. 151, 128-134.

Ma, L., Steinberg, J.L., Cunningham, K.A., Lane, S.D., Bjork, J.M., Neelakantan, H., Price, A.E., Narayana, P.A., Kosten, T.R., Bechara, A., Moeller, F.G., 2015b. Inhibitory behavioral control: A stochastic dynamic causal modeling study comparing cocaine dependent subjects and controls. NeuroImage Clin. 7, 837-847.

McLellan, A.T., Kushner, H., Metzger, D., Peters, R., Smith, I., Grissom, G., Pettinati, H., Argeriou, M., 1992. The fifth edition of the addiction severity index. J. Subst. Abuse Treat. 9, 199-213.

Moeller, F.G., Hasan, K.M., Steinberg, J.L., Kramer, L.A., Dougherty, D.M., Santos, R.M., Valdes, I., Swann, A.C., Barratt, E.S., Narayana, P.A., 2005. Reduced anterior corpus callosum white matter integrity is related to increased impulsivity and reduced discriminability in cocaine-dependent subjects: diffusion tensor imaging. Neuropsychopharmacology 30, 610-617.

Moeller, F.G., Hasan, K.M., Steinberg, J.L., Kramer, L.A., Valdes, I., Lai, L.Y., Swann, A.C., Narayana, P.A., 2007. Diffusion tensor imaging eigenvalues: preliminary evidence for altered myelin in cocaine dependence. Psychiatry Res. 154, 253-258.

Mori, S., Zhang, J.Y., 2006. Principles of diffusion tensor imaging and its applications to basic neuroscience research. Neuron 51, 527-539.

Mori, S., Wakana, S., Van Zijl, P.C.M., 2005. MRI atlas of human white matter. Am. J. Neurorad. 27, 1384-1385.

Murugavel, M., Cubon, V., Putukian, M., Echemendia, R., Cabrera, J., Osherson, D., Dettwiler, A., 2014. A longitudinal diffusion tensor imaging study assessing white matter fiber tracts after sports-related concussion. J. Neurotraum. 31 , 1860-1871.

Nakamura-Palacios, E.M., Lopes, I.B., Souza, R.A., Klauss, J., Batista, E.K., Conti, C.L., Moscon, J.A., de Souza, R.S., 2016. Ventral medial prefrontal cortex (vmPFC) as a 
target of the dorsolateral prefrontal modulation by transcranial direct current stimulation (tDCS) in drug addiction. J. Neural Transm. 123, 1179-1194.

Narayana, P.A., Ahobila-Vajjula, P., Ramu, J., Herrera, J., Steinberg, J.L., Moeller, F.G., 2009. Diffusion tensor imaging of cocaine-treated rodents. Psychiatry Res. 71 242-251.

Narayana, P.A., Herrera, J.J., Bockhorst, K.H., Esparza-Coss, E., Xia, Y., Steinberg, J.L., Moeller, F.G., 2014. Chronic cocaine administration causes extensive white matter damage in brain: diffusion tensor imaging and immunohistochemistry studies. Psychiatry Res. 221, 220-230.

Pfefferbaum, A., Adalsteinsson, E., Sullivan, E.V., 2003. Replicability of diffusion tensor imaging measurements of fractional anisotropy and trace in brain. J. Magn. Reson. Imag. 18, 427-433.

Romero, M.J., Asensio, S., Palau, C., Sanchez, A., Romero, F.J., 2010. Cocaine addiction: diffusion tensor imaging study of the inferior frontal and anterior cingulate white matter. Psychiatry Res. 181, 57-63.

Salimi-Khorshidi, G., Smith, S.M., Nichols, T.E., 2011. Adjusting the effect of nonstationarity in cluster-based and TFCE inference. Neuroimage 54, 2006-2019.

Schmitz, J.M., Green, C.E., Stotts, A.L., Lindsay, J.A., Rathnayaka, N.S., Grabowski, J., Moeller, F.G., 2014. A two-phased screening paradigm for evaluating candidate medications for cocaine cessation or relapse prevention Modafinil, levodopa-carbidopa, naltrexone. Drug Alcohol Depend. 136, 100-107.

Smith, S.M., Nichols, T.E., 2009. Threshold-free cluster enhancement: addressing problems of smoothing, threshold dependence and localisation in cluster inference. Neuroimage 44, 83-98.
Smith, S.M., Jenkinson, M., Johansen-Berg, H., Rueckert, D., Nichols, T.E., Mackay, C.E., Watkins, K.E., Ciccarelli, O., Cader, M.Z., Matthews, P.M., Behrens, T.E., 2006. Tract-based spatial statistics: voxelwise analysis of multi-subject diffusion data. Neuroimage 31, 1487-1505.

Smith, S.M., 2002. Fast robust automated brain extraction. Hum. Brain Mapp. 17, $143-155$.

Taber, K.H., Pierpaoli, C., Rose, S.E., Rugg-Gunn, F.J., Chalk, J.B., Jones, D.K., Hurley, R.A., 2002. The future for diffusion tensor imaging in neuropsychiatry. J. Neuropsychiatry Clin. Neurosci. 14, 1-5.

Tang, Z.C., Dong, E.Q., Liu, J.J., Liu, Z.Y., Wei, W.J., Wang, B., Li, H.J., Tian, J., 2016 Longitudinal assessment of fractional anisotropy alterations caused by simian immunodeficiency virus infection: a preliminary diffusion tensor imaging study. J. Neurovirol. 22, 231-239.

Wakana, S., Caprihan, A., Panzenboeck, M.M., Fallon, J.H., Perry, M., Gollub, R.L. Hua, K., Zhang, J., Jiang, H., Dubey, P., Blitz, A., van Zijl, P., Mori, S., 2007. Reproducibility of quantitative tractography methods applied to cerebral white matter. Neuroimage 36, 630-644.

Xu, J., DeVito, E.E., Worhunsky, P.D., Carroll, K.M., Rounsaville, B.J., Potenza, M.N., 2010. White matter integrity is associated with treatment outcome measures in cocaine dependence. Neuropsychopharmacology 35, 1541-1549.

Yip, S.W., Morie, K.P., Xu, J., Constable, R.T., Malison, R.T., Carroll, K.M., Potenza, M.N., 2016. Shared Microstructural Features of Behavioral and Substance Addictions Revealed in Areas of Crossing Fibers. Biological Psychiatry. CNNI. 